\title{
TASK-RELATED LUMINANCE DISTRIBUTIONS FOR OFFICE LIGHTING SCENARIOS
}

\author{
Sebastian Babilon, Janika Lenz, Sebastian Beck, Paul Myland, \\ Julian Klabes, Stefan Klir, and Tran Quoc Khanh \\ Laboratory of Lighting Technology, Technische Universität Darmstadt, Darmstadt, Germany \\ E-mail: babilon@lichttechnik.tu-darmstadt.de
}

\begin{abstract}
For the design of modern office environments, lighting is a central aspect. With regard to current practice, uniform illumination is most often applied in interiors. In this paper, however, further aspects of a more individual approach are investigated, that deliberately violate the usual demands for uniformity by explicitly considering task-related, emotional and psychological effects of lighting. For this purpose, two independent experiments were conducted in an office mock-up setting exploring the impact of spatially variable, non-uniform light distributions on the users' illumination preferences for the accomplishment of a given task. In the first experiment, three predefined illumination settings were rated by a group of naïve observers. Although the respective light distributions differed in their spatial characteristics, no significant differences were found in the rating scores. In addition, these variations showed no significant effect on the users' preferred position of task performance. In the second experiment, though, a clearly significant effect could be reported such that, once the users were granted control over the illumination settings, an explicit demand for locally increased illuminance levels at the position of task performance was observed. Furthermore, high rating scores of perceived lighting adequacy indicate the users' general satisfaction with the degree of visual assistance provided by such a task-related illumination.
\end{abstract}

Keywords: office lighting, task-related light distributions, user preference, visual comfort, room perception

\section{INTRODUCTION}

Regarding human factors and ergonomics, achieving a sufficient level of lighting quality is an essential requirement for the design of modern office environments, which, as supported by the literature, can contribute to a sustainable increase in environmental satisfaction and individual performance [1-3]. In this context, task visibility, colour appearance, visual comfort, object modelling, and room appearance have been identified to be the most relevant quality measures for functional office lighting [4]. Being composed of all these rather subjective aspects, lighting quality in general is determined by the degree of compliance of the visual conditions with the task-related, emotional, and physiological needs of the office workers, i.e., the more supportive the lit environment is experienced by the individual for the accomplishment of a given task, the higher the respective lighting quality ratings.

This cognitively and emotionally provoked feeling of visual support created by the lighting condition is influenced by various external and user-specific factors, see reference [5] for an extensive overview. From a lighting engineering point of view, only three of these factors are related to the lighting system itself and can therefore explicitly be manipulated in order to dynamically adapt the per- 
ceived lighting condition to the user's task-dependent needs:

- Light intensity, which is closely related to the perceptual attributes of perceived brightness $[6,7]$ and visual clarity [8-10];

- Spectral power distribution, which determines the white point chromaticity [11] and correlated colour temperature (CCT) $[12,13]$ of the room illumination as well as the objects' perceived colour appearance $[14,15]$;

- Spatial light distribution [16-23], which basically summarizes the resulting luminance pattern of all room surfaces given by the luminous intensity distributions of the luminaires.

In recent years, luminaires and lighting solutions have emerged on the market that, besides showing a certain customizability and intelligence regarding their proper adjustment to the desired values of light intensity and spectral composition, also allow for user-controlled directional variations of their luminous intensity distributions. Along with these sophisticated systems comes a new dimension of complexity and freedom in dynamic lighting design. In the past, studies investigating the effect of variations in spatial light distribution on room perception, comfort, preference, health, well-being, or cognitive performance of office workers either focussed on experiments that systematically altered the proportion of the direct and indirect components of suspended luminaires $[17,20,21]$ or examined the potential benefits related to the installation of additional static luminaires, such as sconces, wall washers, uplighters, or spotlights, intended to provide a variable and more appealing room luminance pattern $[16,18,19,22,23]$.

None of these studies, however, included such highly-integrated luminaires that allow for a dynamic, usage-specific and task-related optimization of their individual luminous intensity distributions with regard to the user's needs. In two independent experiments, this work therefore investigates how directional variations in the spatial light distribution defining the luminance pattern on the working plane may affect office workers in the completion of a certain task while the remaining room luminance pattern is kept fixed.

As a future application example, one can think of a single lighting installation that is capable of providing both a task-specific desk illumination temporally increasing local illuminance levels for the individual office worker whenever needed (e.g., during writing and reading exercises) and a generally preferred ambient illumination with a perceptually optimal luminance distribution on the walls and the ceiling as well as a favoured direct/indirect lighting ratio (further research is still necessary to define these "optimal" conditions, see e.g. Sullivan and Donn [24] for review). The flexibility offered by such smart, multi-directional, highly-integrated lighting systems and luminaires may also allow for more sophisticated dimming strategies in the context of occupancy-based lighting control in shared or even open-plan offices to increase energy savings without degrading users' comfort and well-being.

The current paper is organized as follows. In Sec. 2, the conceptual designs and test protocols as used in the current work are discussed for both experiments. The corresponding data analysis including a discussion of its implications is then provided in Sec. 3. A summary of the study's main results and findings is eventually given in Sec. 4 .

\section{EXPERIMENTAL METHOD AND TEST PROCEDURE}

Two independent experiments have been conducted under laboratory conditions using two different office mock-ups to explore the users' preference for task-related spatial light settings. Variable luminance distributions provided by highly-integrated test luminaires enabled the creation of locally increased illuminance levels on the working plane at otherwise fixed room illumination conditions. Details on the conceptual design for both experiments, the test procedure and the study participants will be given in the following.

\subsection{Experiment I}

For the first experiment, a climate-controlled, white-painted model room of $2.40 \mathrm{~m} \mathrm{x} 3.10 \mathrm{~m} \mathrm{x}$ $2.55 \mathrm{~m}$ was equipped with a single white office desk of $1.20 \mathrm{~m} \times 0.60 \mathrm{~m} \times 0.76 \mathrm{~m}$. In order to create the impression of an ordinary visual display unit (VDU) workstation, a computer monitor, mouse, and keyboard, some stacked books, a filled pencil cup, and a small green indoor plant were placed on top of it. The dropped ceiling was kept in white showing the same reflectance as the walls of approximately 0.8 . The greyish matt linoleum with an average reflectance factor of 0.4 covered the floor. Blinds were 

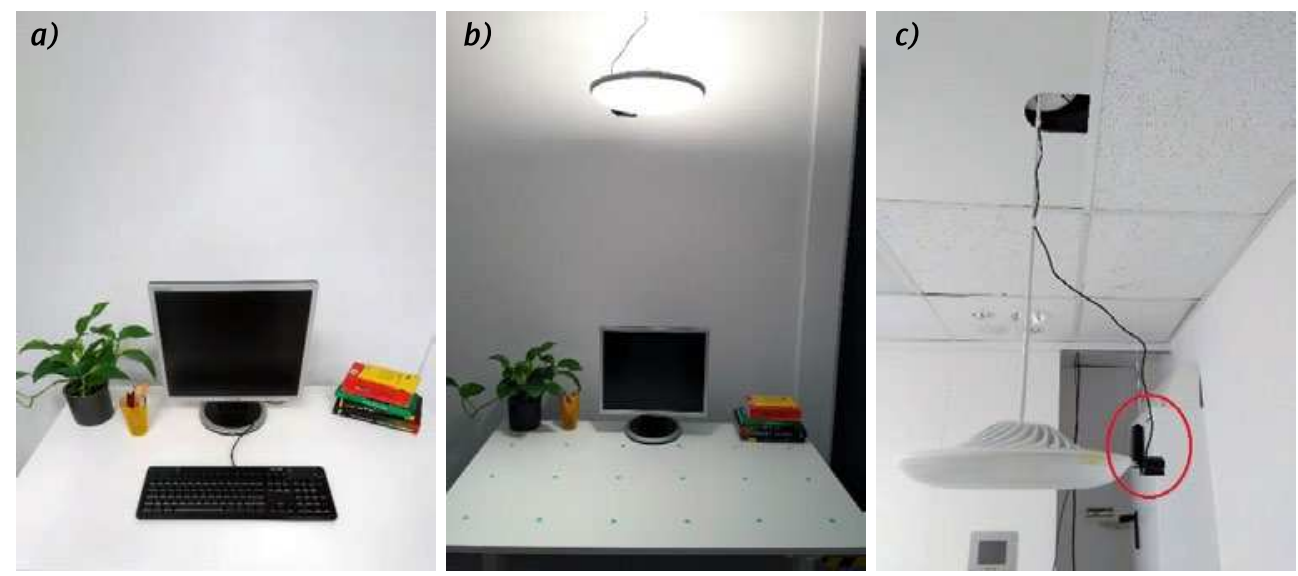

Fig. 1. Image representations of the model room and setup of the first experiment:

(a) Approximate view from the observers' perspective when assessing the different lighting situations the objects placed on top of the office desk create the impression of a VDU workstation and a corresponding working atmosphere;

(b) Test luminaire suspended from the ceiling and aligned to the centre of the office desk and its individual LED segments, both in up- and downlight, can be controlled independently, wherein blue spots temporarily affixed to the surface of the office desk mark the measurement grid for the characterization of the different lighting situations and were removed before starting the actual study;

(c) Camera system used for detecting the position of task performance of the individual subject - the system is installed and adjusted in such a way that it captures the complete surface of the office desk without perceptually interfering with the room luminance pattern or the subjects' view

used to prevent any natural daylight from entering. The computer monitor served solely as a prop and was therefore kept in an off state all the time. The main source of illumination was provided by a multidirectional test luminaire, a Luke Roberts Model $\mathrm{F}$ [25], which was aligned to the centre of the office desk and suspended at a distance of $70 \mathrm{~cm}$ from the ceiling. For a better visualization, Fig. 1 represents a selection of images depicting the experimental room with some further details on the setup.

For conducting the experiments, both up- and downlight of the test luminaire were adjusted to $4000 \mathrm{~K}$ at a maximum CRI value. In order to create an appealing overall room appearance that is largely independent of the variable downlight settings, the up light was energised to maximum light output providing a decently indirect ambient illumination. The peripheral room appearance was additionally determined by the emission of four recessed $4000 \mathrm{~K}$ fluorescent tube luminaires, which were installed into the ceiling behind the observer and dimmed in such a way that an approximately equal illuminance $(\sim 80 \mathrm{~lx})$ and brightness perception could be achieved on all four walls.

Based on this initial lighting condition, three different illumination settings of the test luminaire's downlight were defined:

- Homogeneous (reference) illumination of the office desk;
- Illumination spot on the left;

- Illumination spot on the right.

The characterization of the various lighting situations was performed based on a well-defined measurement grid (see Fig. 1(b); the distance between two measurement points was $20 \mathrm{~cm}$, while $10 \mathrm{~cm}$ were chosen for the distance between the most outer points and the desk edges) by using two calibrated Gigahertz-Optik HCT-99D photometers and a calibrated Gigahertz-Optik X1 $1_{1}$ optometer with four measurement heads. To prevent that one situation is preferred over another just because of different overall illuminance levels on the office desk the emitted downlight intensities for the three lighting conditions were adjusted accordingly. The goal was to reduce the measured average illuminance differences to an absolute minimum. Trial and error eventually yielded an optimal constellation of settings showing a mean average value of $(305 \pm 3) 1 \mathrm{x}$ for the three lighting conditions. The corresponding uniformities were subsequently calculated according to DIN EN12464-1 using the formula

$$
U_{0}=E_{\min } / \bar{E}
$$

where $E_{\min }$ denotes the minimum and $\bar{E}$ the average measured illuminance values. While for the reference a uniformity of 0.79 could be obtained, it was significantly reduced for the two different spot- 

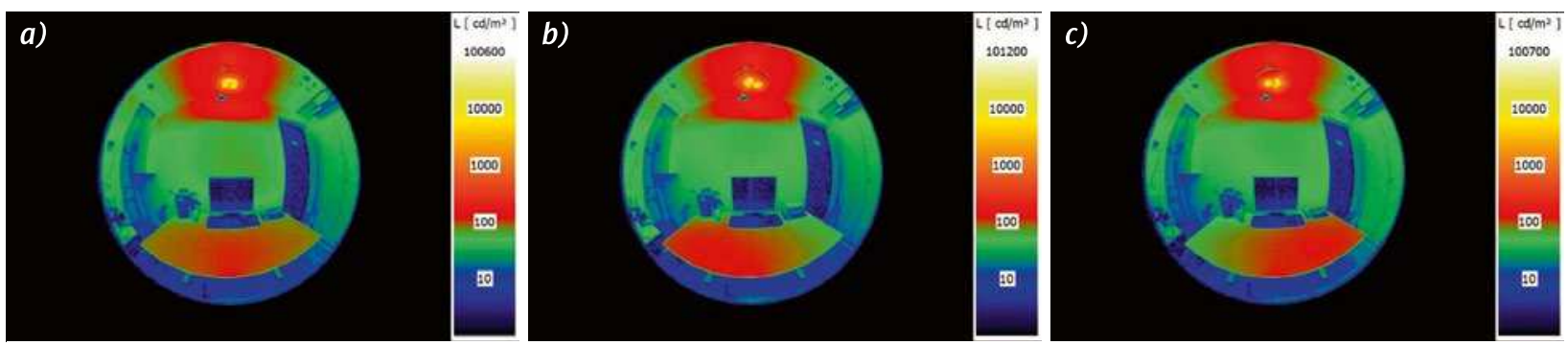

Fig. 2. Visualization of the luminance distributions of the three different lighting situations optimized for the first experiment (the general room appearance is largely independent of the variable downlight settings, variations are only observed

on the office desk due to locally increased illuminance values); the following settings were defined: (a) Homogeneous

(reference) illumination, (b) Illumination spot on the left, and (c) Illumination spot on the right (in each case, an approximately equal average illuminance was ensured to be measured on the office desk)

light situations, showing values of 0.52 (spot on the right) and 0.53 (spot on the left), respectively. For the sake of completeness, Fig. 2 depicts the corresponding luminance distributions as captured by a TechnoTeam LMK 5-1 colour.

A within-subjects study design was chosen for the first experiment with illumination condition as the independent variable. The question to be considered in this context is whether the local illuminance variations, that were limited to the office desk only, were experienced by the study participants and, if so, whether these variations were considered as bothering. For this purpose, the test protocol shown in Fig. 3 was applied. On arrival at the institute, participants were guided to a white-painted anteroom (also climate-controlled) of $3.00 \mathrm{~m} \times 3.10 \mathrm{~m} \times$ $2.55 \mathrm{~m}$, which was equipped with four of the same recessed $4000 \mathrm{~K}$ fluorescent tube luminaires as installed in the adjacent model office providing ho- mogeneous illumination. A single white office desk of $1.60 \mathrm{~m} \times 0.80 \mathrm{~m} \times 0.78 \mathrm{~m}$ was positioned in the middle of the room. Floor and ceiling were of the same kind as in the model office. Again, natural daylight was blocked from entering by using suitable blinds. The horizontal illuminance measured on the desk's surface was also set to 305 lx. During the subsequent $5 \mathrm{~min}$ of adaptation, each participant was asked to complete a short questionnaire assessing age, gender, and handedness and to rate their subjective sleepiness level on the Karolinska Sleepiness Scale [26] (KSS). Next, they were handed over a first standardized C-Test [27] to be completed under one of the three different experimental conditions while being seated at the office desk in the model room. According to their preference, participants could choose between versions in German or English language. Once having received the $\mathrm{C}$-Test sheet, they were requested to enter the mod-
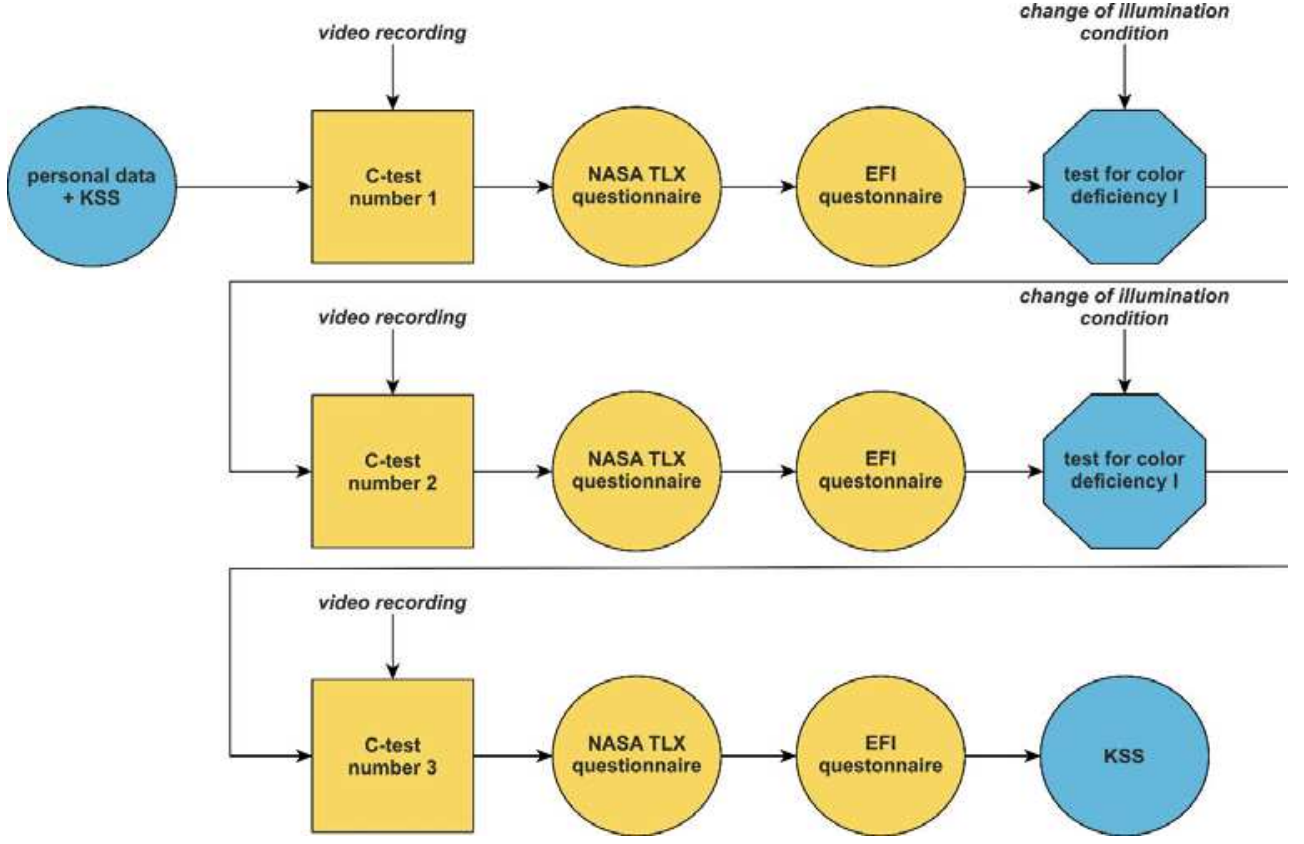

Fig. 3. Test protocol of the first experiment for the assessment of the three different desk illumination settings shown in Fig. 2 (a randomized, withinsubjects study design was chosen, taking the illumination condition as the independent variable, and, in addition, observers were blinded such that they were not consciously aware of the change in illumination that occurred at the end of each experimental round) 


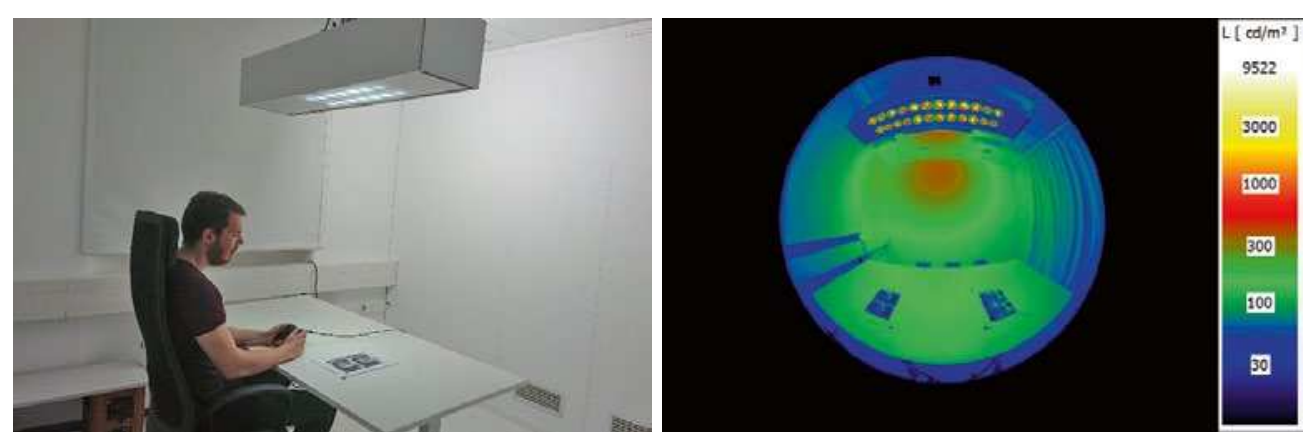

Fig. 4. Experimental setup used for the second experiment for directional lighting, locally increasing the illuminance level on the office desk, was realized by a custom-made test luminaire enabling the adjustment of its luminous intensity distribution (ambient illumination was provided by a soft-light LED panel, which was hidden behind the suspended test luminaire and pointed towards the white opposing wall defining the luminance distribution in the observers' peripheral field of view): (a) with the help of an Xbox 360 controller, participants were able to adjust the local dimming of the illumination of the office desk according to their preference for task performance; (b) reference condition of homogeneous desk illumination

Note to Fig. 4: The task sheet's location on the table was predetermined for each participant by randomly selecting one of the two shown fixed positioning options.

el office on their own. Sitting down at the desk, participants were asked to immediately start with the test. The test sheet's position on the working plane was continuously recorded using the camera system shown in Fig. 1(c).

After having finished the C-Test, which took approximately $5 \mathrm{~min}$ on average, the video recording stopped and two further questionnaires had to be completed. One of which was the NASA Task Load Index [28] (NASA-TLX) questionnaire that assesses the participants' perceived workload during the C-Test exercise, the other an adapted version of the multidimensional questionnaire developed by the Expert Forum Indoor Lighting [29] (EFI) of the German Lighting Society to qualitatively evaluate how an indoor lighting situation is perceived and experienced by the user. Regarding the latter, only those questions that addressed one of the following aspects were adopted from the original EFI questionnaire: - Room and brightness perception;

- Uniformity of the light distribution;

- Potential glare;

- Overall satisfaction with the lighting situation.

After the completion of all tests and questionnaires of the first round, the participants were asked to leave the model office and go back to the anteroom, where they were tested for colour deficiency using the Ishihara Test for Colour Deficiency [30] and the Standard Pseudoisochromatic Plates Part II for Acquired Colour Vision Defects by Ichikawa et al [31]. In the meantime, the lighting situation in the model office was changed to the next setting. Thus, the reason for testing for colour deficiency was twofold such that normal colour vision had to be ensured for all participants to exclude potentially confounding effects and the change of the lighting situation could occur without being noticed.

The test procedure of the first round was also repeated for the second and third with the difference that at the end of the second round a Farnsworth-Munsell D-15 Colour Vision Test [32] was used to check for colour deficiency, while the third round concluded with a second KSS assessment marking the end of the first experiment. To prevent any bias in the assessment of the different lighting situations, the individual settings were presented to each participant in a randomized order.

\subsection{Experiment II}

The second experiment was conducted in the anteroom of the first one. The fluorescent tubes were replaced by a custom-made test luminaire consisting of two Eurolite LED PIX-12 HCL light bars that were joined together alongside each other using a specifically designed frame construction. A single light bar was exactly $1.00 \mathrm{~m}$ of length and contained 12 individually addressable, equidistant multi-channel LED spots, each of which comprised a total number of six different channels (red, green, blue, amber, cool-white, and violet). In order to conceal the individual LED spots from the observer and to create a somewhat smoother overall light distribution, a satin PMMA light diffuser plate was used to cover both light bars. 
The test luminaire was subsequently suspended from the ceiling and aligned to the centre of the office desk. It was fixed to a truss hidden behind the ceiling tiles. The distance between the office desk working plane and the light emitting surface of the luminaire was $1.40 \mathrm{~m}$. Despite the use of a light diffuser plate, interfering colour fringing due to the multi-channel nature of the individual LED spots could be perceived at this distance when driving different channels at the same time. Thus, for conducting the experiments, the LED control was limited to adjusting the amount of light emitted by the cold-white channel only, showing a CRI value of 87 at $5600 \mathrm{~K}$. Similar to the test conditions of Experiment I, an additional light source of the same CCT, in this case a soft-light LED panel of $64.5 \mathrm{~cm} \times 30$ $\mathrm{cm}$ (ARRI SkyPanel S60-C) mounted next to the test luminaire, was used to supplement the local illumination of the office desk by some ambient illumination to create a more spacious and appealing general room appearance. This was obtained by pointing the panel's light emitting surface towards the white opposing wall defining the luminance distribution in the participants' peripheral field of view. Fig. 4 illustrates the general setup and the corresponding luminance distribution for the case that all LED spots of the test luminaire were driven at $35 \%$ of their maximum PWM value.

In total, 16 different illumination settings were defined. In addition to the reference condition of homogeneous desk illumination shown in Fig. 4 (b), directional lighting, locally increasing the illuminance level on the office desk, was provided by sequentially turning pairs of LED spots on and off as depicted in Fig. 4 (a). In this way, it was possible for the user to smoothly shift a perceivable illumination spot from the left side of the desk to the right side by using the upper shoulder buttons of a tethered Xbox 360 controller. In each case, the amount of light emitted by the test luminaire was adjusted such that an average illuminance of $650 \mathrm{~lx}$ was achieved for all illumination settings. This higher-than-standard level was chosen to harmonize the brightness perception between the office desk surface and the opposing wall for an appealing overall room appearance. Illuminance uniformity determined for the working plane was of the order of 0.74 for the homogenous reference and 0.53 for the directional lighting settings. Characterization was again performed using the same measurement grid and calibrated photometers as adopted for the first experiment's setup.

A between-subjects study design on categorical data was chosen for the second experiment. The corresponding test protocol is depicted in Fig. 5. On arrival at the institute, participants were guided to the model office. They were seated at the office desk with the illumination being set to the reference condition. Before starting with the actual experiment, an initial adaptation of $5 \mathrm{~min}$ was required. During this adaptation process, participants were screened for colour deficiency and invited to complete the same personal data questionnaire as adopted for the first experiment. Afterwards, a short introduction was given explaining the test protocol and the use of the controller to adjust the luminous intensity distribution of the test luminaire. After a short training phase, in which participants could switch through all 16 lighting conditions to experience how the local illuminance spot can be shifted and adjusted to different positions and how this influenced their visual perception in relation to the homogeneous reference, the actual experiment started.

In two consecutive walkthroughs, participants were asked to adjust the local dimming of the (directional) illumination of the working plane according to their needs and preferences to solve a given pen-and-paper-based problem. Here, the assigned task was to detect and visualize small, inconspicuous differences between two almost identical images printed on a single sheet of paper. The position of the sheet and, therefore, the location of the task performance were predetermined for each individual participant by randomly selecting one of two different fixed positioning options, one situated on the left and the other on the right hand side of the table, see Fig. 4 (a) and (b). Participants were explicitly instructed to keep the sheet in place and not to move it around. Instead, they should adjust the lighting condition for a subjectively perceived optimal visual support to accomplish the given task at this fixed, predetermined position. Once satisfied with their adjustment, the participants confirmed their impression of adequacy of the current light setting by pushing the " $\mathrm{A}$ " button twice, which immediately initiated an acoustic prompt to start with the task processing. In total, they were given three minutes to find as many differences as possible in the image content. 


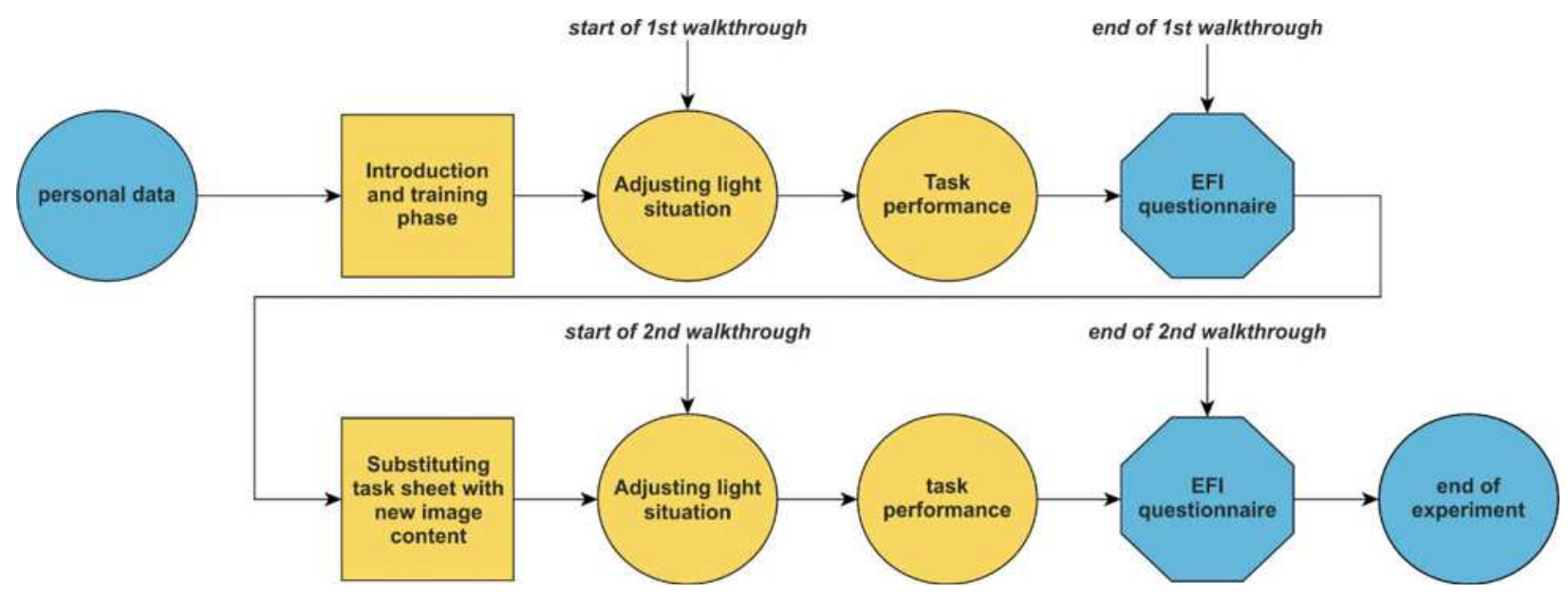

Fig. 5. Test protocol adopted for the second experiment (in two consecutive walkthroughs of adjustment and refinement, participants were asked to adapt the local dimming of the illumination of the office desk to their needs and preferences to experience an optimal visual support for solving the given pen-and-paper-based problem; at the end of each walkthrough, participants should additionally rate the perceived lighting quality of their respective illumination settings)

After these three minutes of task processing, the participants were instructed to put the task sheet aside to their left on a separate small side table and replace it with the EFI questionnaire for a qualitative assessment of the current lighting situation. The completed questionnaire should also be put on the side table, marking the end of the first experimental walkthrough. For the second walkthrough, the participants received a fresh task sheet with new image content that should be placed at exactly the same position as the first one. In contrast to the first walkthrough, where the starting point was always the homogeneous reference, the second walkthrough built upon the participants' previous adjustment. This allowed for an additional refinement in case that the participants on second thought and with the impression gained during the first round of task processing were not a hundred percent satisfied with the current lighting condition. Apart from that, the test protocol of the second walkthrough was identical to those of the first one.

\subsection{Study Participants}

For both experiments, volunteers were recruited through email notices, electronic and conventional postings, and word-of-mouth. They were paid for their participation and only excluded from the study, if they suffered from a known colour vision deficiency or failed any of the colour vision tests performed as part of the respective test protocols. In addition, subjects that had been participating in the first experiment were not considered for taking part in the second. Before being invited to the laboratory, each participant had to sign a declaration of consent approved by the university's ethical review committee. All experiments as well as the data collection and storage were conducted in accordance with national and international ethical standards.

\section{RESULTS AND DISCUSSION}

In the following, regression analysis will be applied to interpret the data collected during the conduct of the first and second experiment. The corresponding results will be reported and discussed in sections 3.1 and 3.2 respectively.

\subsection{Results of the First Experiment}

In total, 30 student volunteers, 19 males and 11 females, completed the first experiment. Three of these subjects were left-handed, the rest right-handed. Their mean \pm standard deviation age was $22.8 \pm$ 2.6 years.

Participants were not informed about the true purpose of the study. Instead, they were invited to take a part in an investigation on the perceived workload as a function of air quality through room-climate regulation. For this reason, the NASA-TLX was included in the test protocol only as a dummy measure and will therefore be excluded from the data analysis. In addition, the subjects' performance on the C-Tests was not of interest for the analysis (in particular since these kind of tests are not suited for assessing work productivity relat- 


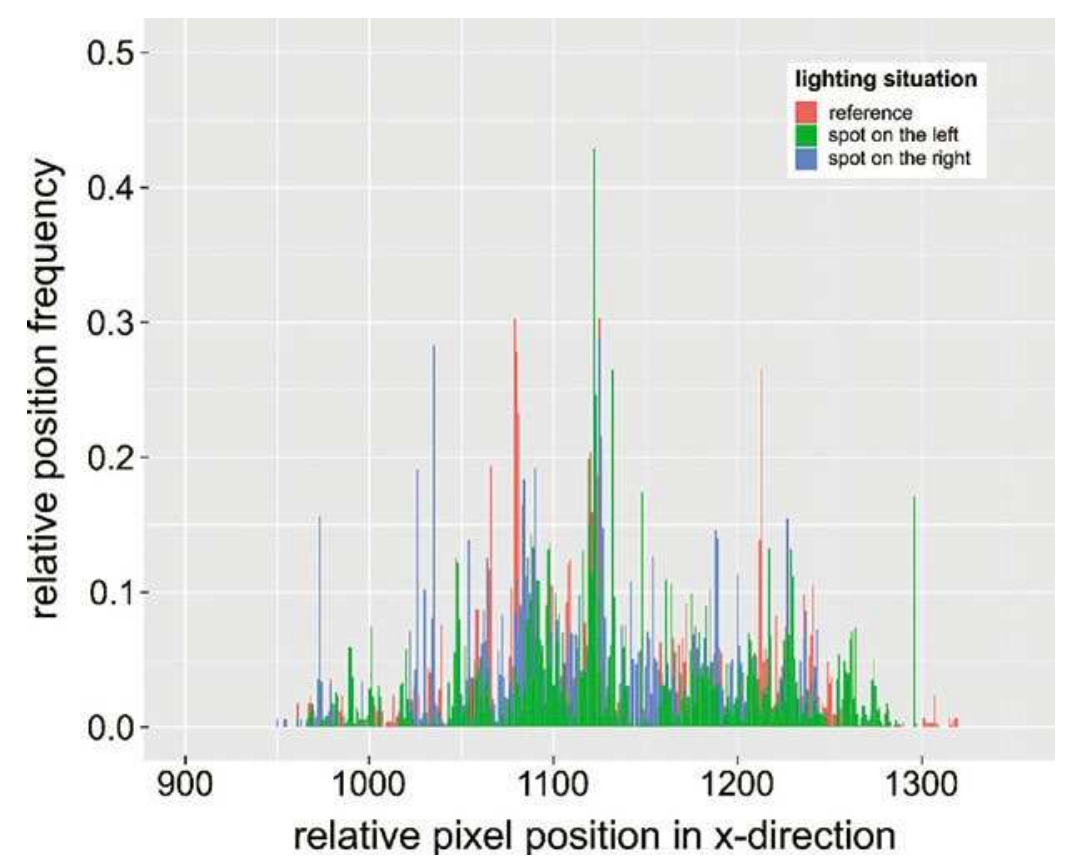

Fig. 6. Relative position frequency of the test sheets' centre coordinates (only x coordinate is of interest) obtained by pooling the video-extracted data of all participants for the three different lighting situations (lower pixel values indicate a tendency to the left, higher pixel values to the right; the results for the homogeneous reference condition (red) are compared to the results of both directional test conditions, i.e desk illumination with spot on the left (green) and on the right (blue), respectively).

Note to Fig. 6: The camera's field of view and, therefore, the corresponding pixel matrix including its origin, does not align with the desk edges, however, since we are only interested in relative differences between the different test conditions and with the camera being held in fixed position, further calibration of the camera system is not required for data analysis

ed to lighting as they are intended to measure language skills only). Yet, they were used to provide a well-defined task to be completed under the different lighting conditions. A corner detection method based on the Harris-Stephens algorithm [33] applied to the video recording data was implemented to determine the subjects' preferred working position as given by the test sheet's centre coordinates in relation to the coordinates of the office desk. For each lighting situation, it was counted frame-by-frame how often the test sheet remained in a certain position. Due to the variable time needed by each participant to complete the $\mathrm{C}$-Test and a constant frame rate of video recording, normalisation was required to pool the data of different observers. Thus, for each participant and lighting situation, the relative position frequency distribution, defined as the number of frames that the test sheet remained in a certain position divided by the corresponding overall number of frames, was calculated.

Fig. 6 depicts the resulting frequency distributions of the three different test conditions as obtained by pooling the data of all participants. Since the luminance pattern on the office desk varied only along the $\mathrm{x}$-axis (Fig. 2), effects on the participants' preferred working position caused by the different lighting conditions are expected to be observed along this direction so that the data analysis can be simplified accordingly. Based on the relative position frequencies extracted from video recording, a weighted average pixel position was subsequently calculated for each participant and lighting condition. Fig. 7 summarizes the corresponding results illustrating potential interactions between lighting condition and gender as well as between lighting condition and handedness.

A linear mixed-effects model (LMM) approach was applied for statistical analysis, where "participant" was entered as a random factor and "lighting condition", "handedness" and "gender" were entered as fixed factors. The analysis revealed no significant main effect of the lighting condition, $\chi^{2}(2)=3.33, p=0.189$, on the participants' weighted average pixel position of their preferred working location on the office desk. There was no noteworthy difference in the pixel positions between left- and right-hander, $\chi^{2}(1)=2.50$ , $p=0.114$, and between female and male participants, $\chi^{2}(1)=0.28, p=0.595$. None of the interaction terms was found to be significant. Thus, the 


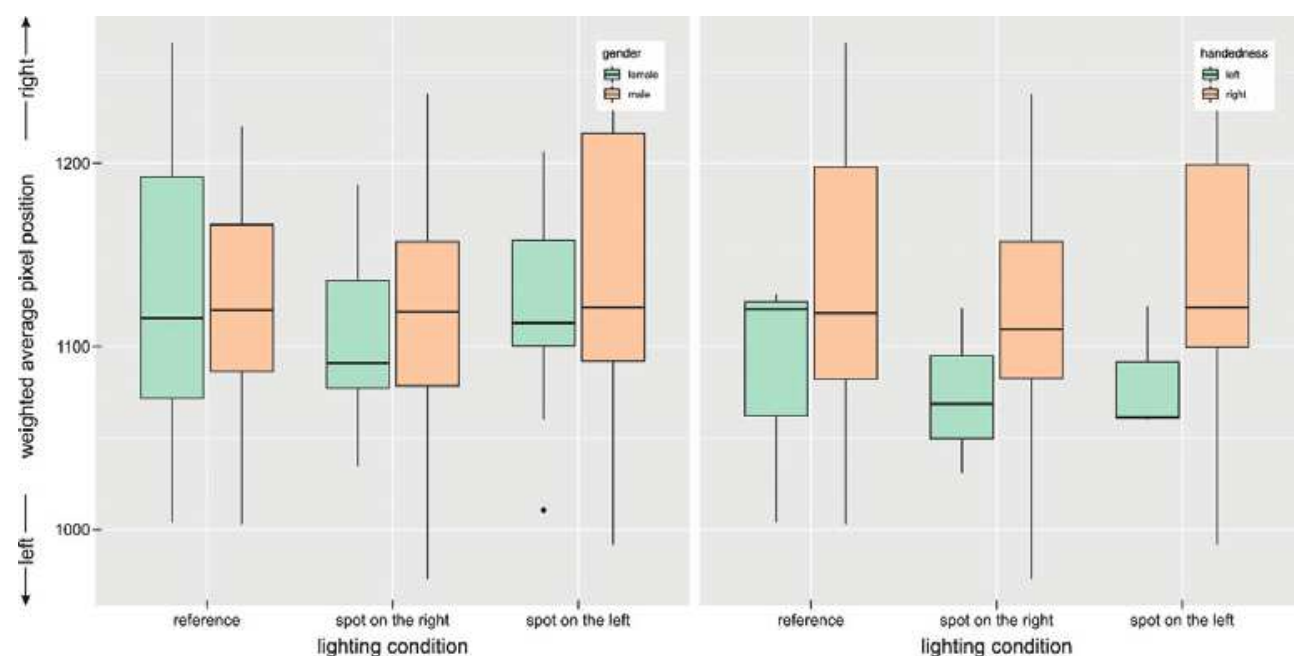

Fig. 7. Boxplots comparing the participants weighted average pixel positions as calculated from their corresponding relative position frequencies for the three different lighting conditions: Smaller (larger) values on the ordinate indicate that the participants' preferred location of task performance was biased to the left (right) side of the table, and no significant differences could be reported, neither for the main effect of the lighting condition nor with regard to the grouping variables of "gender" (plot on the left) and "handedness" (plot on the right)

weighted average pixel position across the different lighting conditions was the same for left- and righthander, $\chi^{2}(2)=0.13, p=0.937$, as well as for men and women, $\chi^{2}(2)=0.75, p=0.688$. In addition, the average pixel position of left- and righthander did not differ, independent of their gender, $\chi^{2}(1)=0.50, p=0.478$.

Regarding the assessment of the user-perceived lighting quality, the participants' EFI rating scores did not significantly differ between the three different lighting situations for any of the considered items. Again, no significant effects of handedness or gender were found. Thus, it can be concluded that, despite introducing a certain amount of inhomogeneity, both directional lighting conditions are rated of at least equally good quality in terms of room and brightness perception, light distribution, glare, and user satisfaction compared to the homogeneously illuminated reference, independent of handedness and gender.

On a closer inspection, directional lighting on average shows even slightly better scores on some of the EFI (sub-) scales than the reference. The room for example was perceived 0.3 points more beautiful, 0.2 points less confined, 0.13 points more interesting, and 0.25 points more activating than in the reference condition. The same holds true for the participants' ratings of their overall satisfaction with the current lighting situation. On average, they were 0.4 points more satisfied with the directional desk illumination. In addition, they showed an increased satisfaction with the general room light- ing conditions of 0.23 points for both non-uniform lighting situations when compared to the homogeneous reference.

Regarding the participants' subjective brightness perception, the rating scores for assessing the workplace, the walls, and the total room appearance were approximately the same across the different lighting conditions. In all cases, the average score differences of the participants' corresponding mean ratings between the two directional and the reference illumination were smaller than 0.1 points and, thus, are negligible. Moreover, there was also an equal demand for slightly larger brightness levels for both the workplace and the walls. For the room appearance, on the other hand, somewhat larger deviations are observed. Here, participants demanded a stronger increase in brightness for the homogeneous reference than for the two directional lighting conditions with an average deviation of 0.25 points on the respective rating scale.

Workplace and room luminance pattern are both experienced to be more uniform for the reference condition than for any of the two different directional light settings. On average, workplace and room are rated 0.47 and 0.22 points more uniform when being homogeneously illuminated. In both cases, however, the difference in the participants' demand for more uniformity was negligibly small between the different lighting situations. In addition, the deflection in either extreme direction on the respective rating scales (i.e., significantly more vs. significantly less uniform) was only little pronounced for 


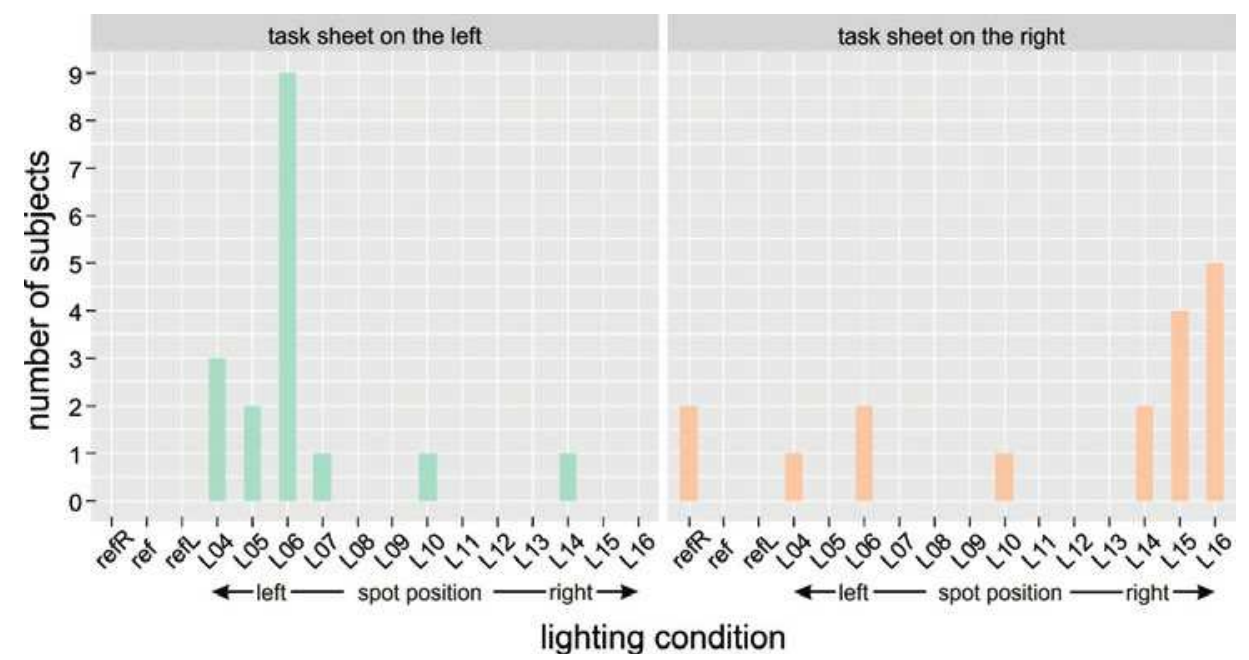

Fig. 8. Number of subjects preferring a certain illumination setting for the case that the task sheet was either placed on the left (plot on the left) or on the right hand side (plot on the right) of the table

Note to the Fig. 8: As illustrated in Fig. 4, participants were able to switch through the different lighting conditions, smoothly shifting the illumination spot on the office desk from its far-left ("L04") to its far-right ("L16") position and vice versa: two intermediate conditions, "refR" and "refL", were additionally created to guarantee a smooth transition between the homogeneous reference condition ("ref") and the more directional light settings of conditions L04 to L16 and a significant association between the task sheet's position on the table and the participants' preferred illumination setting for task performance could be confirmed.

all three illumination settings. This basically indicates that, compared to the reference condition, the participants on average did not feel disturbed by the amount of inhomogeneity introduced in both test conditions.

Moreover, no glare was experienced as portended by the corresponding, relatively high rating scores. In addition, the participants' KSS ratings were always $\leq 5$ and did not significantly differ between the beginning and the end of the test period. This confirms that they all showed a reasonably good level of alertness while taking part in the experiment and that the time of testing was short enough to prevent any fatigue from potentially confounding the reported results.

\subsection{Results of the Second Experiment}

In the second experiment, data of 34 new student volunteers, 28 males and 6 females who were not involved in the first experiment were collected. Seven of these subjects were left-handed, 27 right-handed. Their mean \pm standard deviation age was $25.6 \pm 6.8$ years. For the analysis, we are primarily interested in the participants' preferred illumination settings and corresponding EFI ratings at the end of the second walkthrough's refinement step, so that only these data will be considered in the following. Again, the given task was only in- tended to provide a well-defined framework for assessing task-related user preferences in lighting. Due to its non-standardized, non-validated nature, it is not suited to measure work productivity or to draw any valid conclusions on the subjects' performance level. Corresponding data were therefore excluded from the analysis.

Fig. 8 can then be interpreted as the participants' corresponding preference distribution. It illustrates how often a certain test condition was selected over the others for the accomplishment of the given task. As can be seen, depending on whether the task sheet was positioned on the left or on the right hand side of the table, participants tended to adjust the test luminaire accordingly. Applying a two-tailed Fisher's exact test of independence [34] thus reveals a significant association between the task sheet's position on the table and the participants' illumination preference $(p=0.0012)$. This result confirms the initially formulated hypothesis that, for a perceptually optimal visual support, a directional light situation providing locally increased illuminance levels at the position of task performance is preferred over a homogeneous reference condition - at least in case of pen-and-paper-based work. Again, no significant differences were found with regard to handedness or gender. Thus, in accordance with the results of the first experiment, participants do not really seem to bother about perceptual inhomoge- 
neities that much as long as a general feeling of usage- / task-specific visual assistance is provoked by the illumination conditions.

This conclusion is further supported when looking at the corresponding EFI ratings. Independent of whether the task sheet was located on the left or on the right hand side of the table, the participants' average satisfaction scores after the second walkthrough for both the illumination of the office desk and the general room lighting conditions were 5.45 and 5.33 respectively, which is actually quite good considering that the homogeneous reference of the first experiment on average was rated more than 0.5 points lower on both scales. Again, no glare was perceived and the working plane, the walls, and the room were judged to appear sufficiently bright. What should further be emphasized is that the participants' ratings of the second experiment clearly confirmed the adequacy of a directionally adjusted illumination to support the task fulfilment. This conclusion is based on the scores collected for a new, dedicated item additionally added to the EFI questionnaire: Participants were explicitly asked to rate their feeling of visual support during task performance. The corresponding mean rating after adjusting the lighting conditions was 5.79 for both task sheet positions, indicating that the participants' were generally satisfied with the task-related illumination conditions.

\section{SUMMARY AND OUTLOOK}

In this work, two independent experiments were conducted under laboratory conditions using two different office mock-up settings to explore the impact of spatially variable, non-uniform light distributions on the users' illumination preferences for the accomplishment of a given pen-and-paper-based task. In both experiments, the general room luminance pattern was kept fixed, while changes in illumination were applied to the working plane only. In each case, this was achieved by a combination of standard luminaires defining the ambient conditions and a single, highly-integrated luminaire for the desk illumination with enabled dynamic control of its luminous intensity distribution.

In the first experiment testing naïve observers, a randomized, within-subjects study design was chosen on three predefined light settings - a homogeneous reference and two test conditions with an illumination spot on either the left or the right hand side of the office desk. The goal of this experiment was to qualitatively investigate whether local illuminance variations were experienced by potential office workers during task performance. From the data analysis, no significant differences were found in the respective EFI rating scores of lighting quality. In addition, no significant main effect of lighting condition could be reported with regard to the observers' preferred working location on the office desk. Thus, up to a certain amount of inhomogeneity on the working plane, whose threshold still need to be defined on a future occasion, naïve observers do not seem to be bothered in their task fulfillment by subtle changes of the desk's local illumination.

In the second experiment, participants were explicitly granted control over the illumination's spatial adjustment on the working plane. In this case, a clearly significant effect was observed such that an additional demand for locally increased illuminance levels at the position of task performance could be reported. In addition, the participants' average EFI satisfaction scores after adjustment as well as their corresponding illumination adequacy ratings were quite good, which indicates that a task-related illumination can be beneficial with regard to increasing visual comfort by actively supporting the task fulfilment.

So far, we limited our investigations to simplifying but well-defined pen-and-paper-based tasks performed under controlled laboratory conditions only. With regard to the practical application of the reported results, it would thus be of interest to extend the current study to more realistic, less constrained computer-based office tasks. This brings up the question in what manner the luminance distribution of the computer monitor will interact with the local desk illumination. One could hypothesize that also in this case office workers might prefer a local dimming strategy over the homogeneous illumination reference layout, e.g., by providing an increased illuminance level on the keyboard and mouse but a decreased level in the area of the computer monitor. Upcoming studies investigating the benefits of highly-integrated, multidirectional luminaires in the context of task-related, user-preferred lighting are therefore required and should be conducted in real office environments for an increased practical utility. In addition, future work may further focus on the link between task-optimized lighting conditions and a potential increase in office 
workers' productivity. For this purpose, though, reliable and validated measures for work productivity and/or individual performance improvements must be incorporated accordingly.

Task-related dimming strategies geared to the specific needs of an individual office worker are expected to become more and more important. Besides offering the possibility of increasing the users' visual comfort and well-being in the office environment, as suggested by the results discussed in this paper, they can also help fulfil energy savings requirements, primarily by expanding the concepts of occupancy-based lighting control by a more task-related approach. Due to the increasing flexibility of modern, highly-integrated lighting systems regarding the directions of light emission and LED control, a single lighting installation consisting of a relatively small number of this kind of luminaires may be sufficient for providing both a generally preferred ambient illumination of the office environment and a task-specific desk illumination temporally increasing local illuminance levels whenever needed by the individual office worker. Thus, no additional desk lamps or free-standing luminaires would be required anymore for achieving an increased visual comfort, reducing installation and maintenance costs. In addition, the potential use of artificial intelligence for automatically recognizing the users' needs and adapting the lighting conditions accordingly is also something of huge benefit that should be addressed in the future. The goal must be to ease system control as far as possible and, despite its complexity, ensure sustained user acceptance by preventing the user from feeling overwhelmed by a too large number of adjustment options.

\section{DISCLOSURES}

The authors declare that there were no conflicts of interest related to this article and the underlying research.

\section{ACKNOWLEDGMENTS}

The authors would like to thank the Luke Roberts $\mathrm{GmbH}$ for providing the first experiment's test luminaire, a Luke Roberts Model F including its API, as well as for their much-appreciated support regarding all issues of device control. This research was funded as part of an individual research grant of DFG with project number 445336968.

\section{REFERENCES}

1. Boyce P.R., Veitch J.A., Newsham G.R., Jones C.C., Heerwagen J., Myer M., Hunter C.M. Lighting quality and office work: Two field simulation experiments// Lighting Research and Technology, 2006, \# 38, pp. 191-223.

2. Veitch J.A., Newsham G.R. Lighting quality and energy-efficiency effects on task performance, mood, health, satisfaction, and comfort// Journal of the Illuminating Engineering Society 1998, \# 27, pp. 107-129.

3. Veitch J.A., Newsham G.R., Boyce P.R., Jones C.C., Lighting appraisal, well-being and performance in openplan offices: A linked mechanisms approach// Lighting Research and Technology, 2008, \# 40, pp. 133-151.

4. Knoop M. Lighting quality measures for interior lighting with LED lighting systems// Proceedings of the 27th CIE session, Sun City, South Africa, 2011, pp. 219-225.

5. Khanh T.Q., Bodrogi P., Guo X., Anh P.Q. Towards a user preference model for interior lighting Part 1: Concept of the user preference model and experimental method// Lighting Research and Technology, 2019, \# 51, pp. 1014-1029.

6. Rea M.S., Mou X, Bullough J.D. Scene brightness of illuminated interiors// Lighting Research and Technology, 2016, \# 48, pp. 823-831.

7. Duff J., Kelly K., Cuttle C. Perceived adequacy of illumination, spatial brightness, horizontal illuminance and mean room surface exitance in a small office// Lighting Research and Technology, 2017, \# 49, pp. 133-146.

8. Thornton W.A., Chen E. What is visual clarity// Journal of the Illuminating Engineering Society, 1978, \#7, pp. 85-94.

9. Hashimoto K., Nayatani Y. Visual clarity and feeling of contrast// Color Research and Application, 1994, \# 19, pp. 171-185.

10. Vrabel P.L., Bernecker C.A., Mistrick R.G. Visual performance and visual clarity under electric light sources: Part II-Visual Clarity// Journal of the Illuminating Engineering Society, 1998, \# 27, pp. 29-41.

11. Bodrogi P., Guo X., Stojanovic D., Fischer S., Khanh T.Q. Observer preference for perceived illumination chromaticity// Colour Research and Application, 2018, \# 43, pp. 506-516.

12. Boyce PR, Cuttle C. Effect of correlated colour temperature on the perception of interiors and colour discrimination performance// Lighting Research and Technology, 1990, \# 22, pp. 19-36.

13. Bodrogi P., Khanh T.Q., Stojanovic D., Lin Y. Intercultural colour temperature preference of Chinese and 
European subjects living in Germany// Light and Engineering, 2016, Vol. 24, \# 1, pp. 8-11.

14. Khanh T.Q., Bodrogi P., Vinh Q.T., Stojanovic D. Colour preference, naturalness, vividness and colour quality metrics, Part 2: Experiments in a viewing booth and analysis of the combined dataset// Lighting Research and Technology, 2017, \# 49, pp. 714-726.

15. Khanh T.Q., Bodrogi P., Guo X., Vinh Q.T., Fischer S. Colour preference, naturalness, vividness and colour quality metrics, Part 5: A colour preference experiment at $2000 \mathrm{~lx}$ in a real room// Lighting Research and Technology, 2019, \# 51, pp. 262-279.

16. Flynn J.E., Spencer T.J., Martyniuk O., Hendrick C. Interim study of Procedures for investigating the effect of light on impression and behaviour// Journal of the Illuminating Engineering Society, 1973, \# 3, pp. 87-94.

17. Hentschel H.J., Klein E., Leibig J., Roll K.F. Energy-effective direct/indirect office and VDU-lighting systems: Test and Application// Journal of the Illuminating Engineering Society, 1987, \#16, pp. 89-105.

18. Loe D.L., Mansfield K.P., Rowlands E. Appearance of lit environment and its relevance in lighting design: Experimental study// Lighting Research and Technology, 1994, \# 26, pp. 119-133.

19. Tiller D.K., Veitch J.A. Perceived room brightness: Pilot study on the effect of luminance distribution// Lighting Research and Technology, 1995, \# 27, pp. 93-101.

20. Houser K.W., Tiller D.K., Bernecker C.A., Mistrick R.G. The subjective response to linear fluorescent direct/indirect lighting systems// Lighting Research and Technology, 2002, \#34, pp. 243-264.

21. Fostervold K.I., Nersveen J. Proportions of direct and indirect indoor lighting - The effect on health, wellbeing and cognitive performance of office workers// Lighting Research and Technology, 2008, \# 40, pp. 175-200.

22. de Vries H.J.A., Heynderickx I.E.J., de Kort Y.A.W., de Ruyter B. Wall illumination - Beyond room appraisal// Proceedings of the 28th CIE Session, Manchester, UK, 2015, pp. 284-290.
23. Chraibi S., Crommentuijn L., van Loenen E., Rosemann A. Influence of wall luminance and uniformity on preferred task illuminance// Building and Environment, 2017, \# 117, pp. 24-35.

24. Sullivan J.T., Donn M. Light distribution and spatial brightness: Relative importance of the walls, ceiling, and floor// Proceedings of the CIE2016 Lighting Quality and Energy Efficiency Conference, Melbourne, Australia, 2016, pp. 59-69.

25. Luke Roberts Model F datasheet. https://hessenbox.tu-darmstadt.de/getlink/fiNnePviFYwNPtSBVin3iwi6/Datasheet_Luke_Roberts_Model_F.pdf, last accessed: 2020/07/14.

26. Åkerstedt T., Gillberg M. Subjective and objective sleepiness in the active individual// International Journal of Neuroscience, 1990, \#52, pp. 29-37.

27. Klein-Braley C, Raatz U. A survey of research on the C-Test// Language Testing, 1984, \#1, pp. 134-146.

28. Hart S.G., Staveland L.E. Development of the NASA-TLX (Task Load Index): Results of empirical and theoretical research// Advances in Psychology, 1988, \#52, pp. 139-183.

29. Moosmann C., Vandahl C. LiTG-Fragebogen zur Bewertung von Lichtsituationen// Proceedings of the Lux Junior 2015, Dörnfeld, Germany, 2015.

30. Ishihara S. Ishihara's Tests for Colour Deficiency: 38 Plates Edition. Tokyo, Japan: Kanehara Trading Inc., 2016.

31. Ichikawa H., Hukami K., Tanabe S. Standard Pseudoisochromatic Plates Part II: For Acquired Color Vision Defects. Tokyo, New York: Igaku-Shoin, 1983.

32. Linksz A. The Farnsworth panel D-15 test// American Journal of Ophthalmology, 1966, \# 62, pp. 27-37.

33. Harris C., Stephens M. A combined corner and edge detector// Proceedings of the 4th Alvey Vision Conference, Manchester, UK, 1988, pp. 147-151.

34. Fisher R.A. On the interpretation of $\chi^{2}$ from contingency tables, and the calculation of $\mathrm{P} / /$ Journal of the Royal Statistical Society, 1922, \# 85, pp. 87-94.

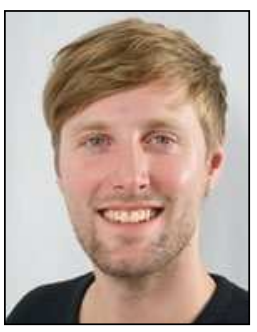

\section{Sebastian Babilon,}

Dr.-Ing., received a doctoral degree in lighting engineering from the Technical University, Darmstadt, Germany, in 2018. Since 2014, he has been working at the Laboratory of Lighting Technology in Darmstadt - now as a Postdoc - focusing on task-related and integrative lighting, memory related colour perception, and the spectral optimization of multi-channel LED luminaires for improved user preference 


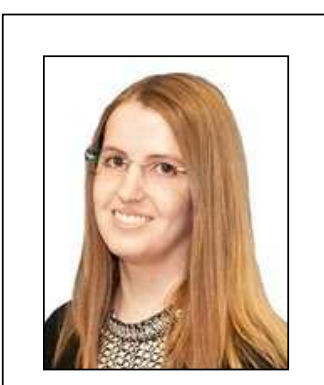

\section{Janika Lenz,}

B. Sc., received her B. Sc. degree in the field of Electrical Engineering and Information Technology from the Technical University of Darmstadt, Germany, in 2019. The current paper reflects the main results of her Bachelor's thesis. Currently, she is pursuing her M. Sc. degree

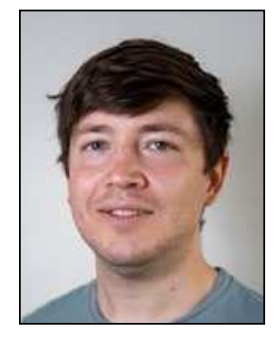

\section{Sebastian Beck,}

M. Sc., received his M. Sc. degree in Information System Technology from the Technical University of Darmstadt, Germany, in 2016. Since then, he has been a research assistant at the Laboratory of Lighting Technology at the Technical University of Darmstadt. His main research areas are integrative lighting, specializing in the optimization of workplace design, and the impact of light on human physiology

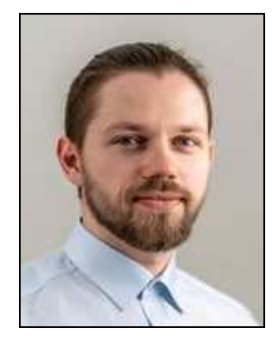

\section{Paul Myland,}

M. Sc., received his M. Sc. degree in the field of Electrical Engineering and Information Technology from the Technical University of Darmstadt, Germany, in 2019. Currently, he is working as a research assistant at the Laboratory of Lighting Technology at TU Darmstadt. His research interests include spectral sensing, colour science, indoor lighting and computer vision

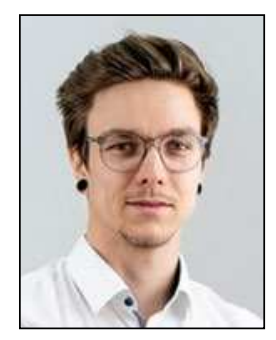

\section{Julian Klabes,}

M. Sc., received his M. Sc. in the field of Electrical and Information Systems from the Technical University of Darmstadt, Germany, in 2019. He is currently completing his Ph.D. at the Laboratory of Lighting Technology of the Technical University of Darmstadt. His main research focus is on empirical methods for the development of lighting quality models in the context of integrative lighting applications

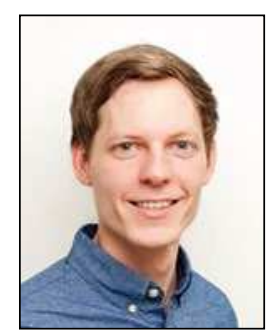

\section{Stefan Klir,}

M. Sc., received his M. Sc. degree in the field of Electrical Engineering and Information Technology from the Technical University of Darmstadt, Germany, in 2017. He is currently pursuing his Ph.D. at the Laboratory of Lighting Technology, Technical University of Darmstadt, Germany. His research is focused on the development of intelligent, deep-learning based lighting systems for integrative lighting applications and increased user preference

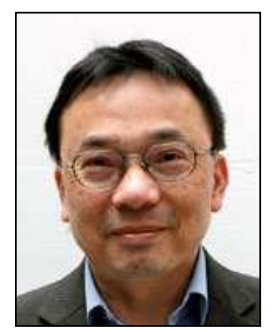

\section{Tran Quoc Khanh,}

Prof. Dr.-Ing. habil., is University Professor and Head of the Laboratory of Lighting Technology at the Technical University of Darmstadt, Germany. He graduated in Optical Technologies and obtained a doctoral degree in lighting engineering from the Technical University of Ilmenau, Germany, in 1989. Before being appointed as a Professor in 2006, he gathered industrial experience as a project manager for photometry, radiometry, and colorimetry at PRC Krochmann and Gigahertz Optik, and later as a technical manager for imaging systems at ARRI. He is the author of several books on lighting technology and related subjects. His research interests cover all important aspects of modern lighting technology including LEDs, colorimetry, integrative lighting, mesopic vision, glare, photometry, and colour science-related topics 